\title{
Debate on MERS-CoV respiratory precautions: surgical mask or N95 respirators?
}

\author{
Jasmine Shimin Chung $^{1}$, MBBS, MRCP, Moi Lin Ling $^{2}$, MBBS, FRCPA, Wing Hong Seto ${ }^{3}$, MRCP, FRCPath,
} Brenda Sze Peng Ang ${ }^{4}$, MBBS, MPH, Paul Anantharajah $\underline{\text { Tambyah }}^{5}$, MD, MBBS

\begin{abstract}
Since the emergence of Middle East respiratory syndrome coronavirus (MERS-CoV) in mid-2012, there has been controversy over the respiratory precaution recommendations in different guidelines from various international bodies. Our understanding of MERS-CoV is still evolving. Current recommendations on infection control practices are heavily influenced by the lessons learnt from severe acute respiratory syndrome. A debate on respiratory precautions for MERS-CoV was organised by Infection Control Association (Singapore) and the Society of Infectious Disease (Singapore). We herein discuss and present the evidence for surgical masks for the protection of healthcare workers from MERS-CoV.
\end{abstract}

Keywords: MERS-CoV, N95 respirators, surgical masks

\section{INTRODUCTION}

Middle East respiratory syndrome coronavirus (MERS-CoV) was first reported in a Saudi Arabian patient in September 2012. Subsequently, MERS-CoV was retrospectively diagnosed in a cluster from Jordan in April 2012. As of 23 April 2014, 253 laboratory-confirmed cases and 93 deaths have been reported to the World Health Organization (WHO), with a reported case fatality rate (CFR) of $36.8 \% .^{(1,2)}$ There has also been an increase in the number of cases of MERS-CoV in Saudi Arabia and the United Arab Emirates (UAE), as reported by WHO between March and April 2014, with healthcare workers among those infected. ${ }^{(3)}$ More recently, there were two reports of MERS-CoV in Southeast Asia. On 17 April 2014, the Ministry of Health Malaysia announced its first and only imported case of MERS$\mathrm{CoV}$ - the death of a Malaysian who had travelled to Saudi Arabia on Umrah pilgrimage - the first death in Southeast Asia. ${ }^{(4)}$ In the Philippines, asymptomatic carriage of MERS-CoV was reported in a Filipino nurse who had been working in the UAE. He was detected through contact screening of a fatal case among a cluster of 11 cases in Abu Dhabi, the UAE. ${ }^{(4)}$

The emergence of these new viruses has raised fears of an epidemic similar to that of severe acute respiratory syndrome (SARS). This is especially so in light of the recent report of fatal MERS-CoV occurring so close to home in neighbouring Malaysia. ${ }^{(4)}$ The threat of a pandemic caused by novel respiratory viruses such as MERS-CoV is real, especially when we have little protective immunity against them. In addition, the potential spread of the novel virus is facilitated by globalisation and international travel, especially as many make their way to Saudi Arabia for the annual Hajj and Umrah pilgrimages, in addition to business travel and adventure trips by students.

As SARS is predominantly a nosocomial infection, a lot of attention was focused on masks for the protection of healthcare workers. There is, however, no consensus among international bodies on the use of droplet or airborne precautions for the prevention of MERS-CoV transmission. WHO, in its May 2013 interim guidance on infection prevention and control during healthcare of cases of probable or confirmed MERS-CoV infection, advocates the use of surgical masks when caring for suspects with possible or confirmed MERS-CoV and particulate respirators for aerosol-generating procedures. ${ }^{(5)}$ This is in contrast to the recommendations of the Health Protection Agency of the United Kingdom (HPA UK) and the United States Centers for Disease Control and Prevention (US CDC), which both advocate the use of particulate respirators and airborne precautions for all patient care activities. ${ }^{(6,7)}$

In response, Infection Control Association (Singapore) and the Society of Infectious Disease (Singapore) organised a debate on MERS-CoV precautions on 20 July 2013. Infectious diseases and infection control experts compared the use of surgical masks versus N95 respirators for MERS-CoV precautions. This paper summarises the proceedings of the event, where the evidence behind MERS-CoV transmission and hospital policies for its prevention were shared.

\section{The debate methodology}

The debate was chaired by Dr Brenda Ang, Senior Consultant, Tan Tock Seng Hospital, and Dr Joanne Tay, Deputy Director, Communicable Diseases Division, Ministry of Health, Singapore. It opened with Dr Ling Moi Lin, Director of Infection Control and Senior Consultant, Singapore General Hospital, proposing the adequacy of surgical masks for the prevention of MERS-CoV transmission. This was followed by Professor Paul Anantharajah Tambyah, Senior Consultant, Division of Infectious Diseases, National University Health System, who advocated the use of N95 masks for MERS-CoV. This was followed by comments from Professor Seto Wing Hong, Chairman of Infection Control, Chief of Service of Microbiology at Queen Mary Hospital and Hong Kong 
West Cluster, Hospital Authority, Hong Kong, and a board member of WHO Collaborating Centre for Infection Control. A series of questions were later posed to a panel of experts consisting of Dr Ling Moi Lin, Professor Paul Anantharajah Tambyah, Professor Seto Wing Hong, Dr Brenda Ang and Dr Joanne Tay.

\section{DISCUSSION}

\section{Surgical mask is adequate - Dr Ling Moi Lin}

Since the emergence of MERS-CoV in September 2012, there have been cases of nosocomial transmission of MERS-CoV in France and eastern Saudi Arabia, but no cases of sustained human-tohuman transmission. ${ }^{(8,9)}$

Guery et al reported the first nosocomial transmission of MERS-CoV - a 51-year-old French national with no prior travel history developed severe MERS-CoV pneumonia after sharing a room with a 64-year-old index patient who had travelled to Dubai. The index patient had presented with atypical symptoms, complaining of fever, chills and diarrhoea. Dyspnoea developed only on Day 4 of hospitalisation. After seven days, MERS-CoV was suspected in the index patient and confirmed by reverse transcription polymerase chain reaction (RT-PCR) on Day 14 of hospitalisation. Despite the delay in diagnosis, and lack of isolation and infection control precautions, there was only one reported transmission to a fellow patient. No secondary transmission was reported in more than 100 healthcare workers who were tested. These healthcare workers had used inconsistent forms of personal protective equipment (PPE). In addition, there were no cases of secondary transmission among the contacts of the second patient, who was eventually discharged. ${ }^{\left({ }^{(8)}\right.}$

In another hospital outbreak in eastern Saudi Arabia, there were 23 confirmed cases and 11 probable cases in healthcare facilities in Al-Hasa (by far the largest nosocomial transmission reported to date). Epidemiology and phylogenetic studies were not able to ascertain single or multiple introductions from the community. In addition, it was not possible to determine the route of transmission from this report or ascertain whether the virus was transmitted when the contact was more than one metre away from the case patient. ${ }^{(9)}$

From the above published reports, MERS-CoV, a beta coronavirus, is more likely to be transmitted through droplets and contact. This is consistent with what we know about other coronaviruses, including the SARS coronavirus. ${ }^{(10,11)}$ Given the phylogenetic and immunologic similarities between MERS-CoV and SARS, infection control precautions could be extrapolated from the experience with SARS in 2003.

Seto et al, in a case control study in five Hong Kong hospitals, showed that the use of masks significantly reduced the risk of SARS infection among healthcare workers. Among noninfected staff members who were surveyed and wore masks, 30.7\% of them had used surgical masks, 54.4\%, N95 masks and 15.3\%, paper masks. Droplet and contact precautions alone were sufficient to protect against SARS. ${ }^{(12)}$

MERS-CoV transmission is most likely to occur when it is not suspected and when lapses or noncompliance with infection control practices occur. Basic infection control measures, including (a) ongoing surveillance programmes; (b) hand hygiene; (c) good environmental hygiene; (d) effective staff health programmes; (e) appropriate outbreak investigation response; (f) education and communication for staff compliance with infection control; (g) sufficient full-time infection control nurses; and (h) having the appropriate infrastructure in place (such as supervision by infection control doctors), are equally important for the prevention of SARS or MERS-CoV transmission as the use of specific PPE. ${ }^{(13)}$

The recommendation for the use of N95 masks may be counterproductive. Its use is often perceived by healthcare workers to be difficult to tolerate, as shown in Toronto during the SARS outbreak. ${ }^{(14)}$ They have also been associated with impaired mental performance and increased headache in healthcare workers, ${ }^{(15-17)}$ which may explain the poor use of N95 masks. For a droplet-transmissible infection, a properly worn surgical mask is more protective than an ill-fitted, inappropriately used N95 mask.

In summary, MERS-CoV is postulated to be a droplet- and contact-transmissible respiratory virus infection with no sustained person-to-person human transmission. Strict adherence to basic infection control measures and proper application of surgical masks is protective, without the need for airborne isolation using N95 respirators.

\section{The case for airborne precautions - Professor Paul Anantharajah Tambyah}

The reports of nosocomial transmission of MERS-CoV and person-to-person transmission within family clusters in the UK and Riyadh, Saudi Arabia, are worrying, for this suggests possible airborne transmission of MERS-CoV..$^{(8,9,18,19)}$ Transmission of MERS$\mathrm{CoV}$ to healthcare workers has also been reported..$^{(9)}$

The phylogenetic and immunologic similarities between SARS and MERS-CoV should not lull us into extrapolating data and experience from SARS in its entirety. ${ }^{(10,11,20)}$ There are stark differences in CFRs at the moment, and hence, the anxiety level of healthcare workers has been raised. Based on WHO's data, the CFR for SARS is relatively lower, ranging from $0 \%$ to $50 \%$, depending on the age group affected, with an overall CFR of $14 \%-15 \% .{ }^{(21)}$ In contrast, the CFR for MERS-CoV is $43.5 \%$ and is as high as $60.0 \%$ in those with comorbidities. ${ }^{(1,22)}$

For a novel respiratory virus that could potentially cause severe disease with reports of transmission to healthcare workers, a good and cost-effective way to prevent its transmission in the healthcare setting is through barrier precautions, which include the use of gloves, gowns and masks. ${ }^{(23)}$ Specifically for respiratory precautions, the use of N95 respirators is recommended for MERSCoV, a novel respiratory virus with a high CFR, especially when N95 respirators are readily available. The use of surgical masks alone may not be sufficient. Our understanding of MERS-CoV is still evolving. Potentially fatal complications, including death, are dire. Healthcare workers need to have the confidence that they are protected, and even if their requests are irrational, these concerns should be considered.

This recommendation is in keeping with international guidelines from HPA UK and US CDC. ${ }^{(6,7)}$ As a developed, 
well-resourced country, we cannot afford to offer our staff any lower level of protection than what is offered in the UK and US.

\section{Trying our best to do what is right - Professor Seto Wing Hong}

The spread of MERS-CoV through travel and tourism, which could result in pandemics with potential adverse impact on the global economy, is always a concern. However, as of 17 July 2013, WHO has unanimously decided that conditions for a public health emergency of international concern for MERS-CoV have not yet been met. In addition, WHO does not advise special screening at points of entry or recommend the application of any trade and travel restrictions. The use of entry temperature screening is controversial, with significant human resource implications and questionable effectiveness.

Pertaining to respiratory precautions, HPA UK and US CDC have recommended airborne precautions. ${ }^{(6,7)} \mathrm{WHO}$ has, however, recommended the use of surgical masks when caring for patients suspected of possible or confirmed MERSCoV and particulate respirators (N95) for aerosol-generating procedures. ${ }^{(5)}$ Recommendations for the use of particulate respirators and airborne precautions during aerosol-generating procedures can be appreciated by extrapolating from the experience with SARS. The highest risk of transmission of SARS to healthcare workers was consistently reported during tracheal intubation, (24-27) and during noninvasive ventilation, tracheotomy and manual ventilation before intubation. ${ }^{(28,29)}$

Recommendations for airborne precautions during routine care of patients with possible or confirmed MERS-CoV are less clear. SARS, which bears a close phylogenetic relationship to MERS-CoV, is spread through droplets and contact. In cases of lower respiratory tract infections, the lungs are congested, making it less likely for aerosol droplets to be generated during normal respiration and coughing. In a systematic review by Jefferson et al in 2010, the use of face masks significantly reduced the spread of respiratory viruses. The same was noted with hand washing and the application of PPE. ${ }^{(23)}$

Recommendations for respiratory precautions in a pandemic setting often evolve with time. This is best illustrated by the 2009 influenza A (H1N1) outbreak. At that time, the Society of Healthcare Epidemiology of America (SHEA) and WHO recommended the use of droplet precautions when caring for patients with suspected or confirmed $\mathrm{H} 1 \mathrm{~N} 1$ based on the understanding that influenza viruses are transmitted by large respiratory droplets. ${ }^{(30,31)}$ US CDC and the Institute of Medicine (IOM), however, recommended the use of N95 in all circumstances in 2009, ${ }^{(32,33)}$ in light of evidence of detectable airborne influenza virus at various locations in a healthcare facility. ${ }^{(34)}$ As the pandemic evolved, and with evidence from a randomised control trial suggesting that surgical masks were as effective as $\mathrm{N} 95$ masks in the prevention of nosocomial pneumonia, ${ }^{(35)}$ US CDC later revised their 2010 guidelines, recommending the use of droplet precautions for confirmed or suspected influenza cases and the use of N95 masks for aerosolgenerating procedures.
Novel viruses with pandemic potential will continue to emerge. Our recommendations for infection control purposes should be evidence-based, and not fuel paranoia and anxiety among healthcare workers.

\section{CONCLUSION}

Every year, millions go on Hajj and Umrah pilgrimages to the Arabian Peninsula, which is the epicentre of the novel virus, MERS-CoV. Upon return, it is common for these pilgrims to present to healthcare facilities with febrile respiratory illnesses. The spread of MERS-CoV with travellers as potential vectors is most feared. Surveillance and infection control strategies are crucial for the containment of MERS-CoV.

There remains no international or regional consensus on the recommendations for respiratory precautions for MERS-CoV among the various infection control professionals. Infection control practitioners at the debate cited their experiences during the SARS epidemic and highlighted the use of a mixture of different masks in Hong Kong and Singapore institutions, with different outcomes. Some healthcare workers exposed to SARS with minimal precautions did not contract the disease, while there were anecdotal reports of those who used the N95 mask and contracted SARS. The reality is that we may never be able to determine the best protective gear to wear for SARS, MERS-CoV or other emerging infections, as it is practically impossible to conduct any randomised controlled trials.

Although evidence presented at the debate supported droplet precautions, except for aerosol-generating procedures, it was evident during the discussion that policymakers would prefer to err on the side of caution and support recommendations for full protective equipment, including the use of N95 masks for MERS$\mathrm{CoV}$, an emerging novel respiratory virus with a potentially high CFR. However, infection control practices and recommendations must be revised as new data emerges. Ongoing surveillance, education, audit of infection control practices and research on the transmission of these novel pathogens are critical and allow us to be in a better position to make practical, safe and appropriate recommendations on infection control practices without fuelling healthcare worker anxiety.

To date, there have been no cases of MERS-CoV in Singapore. However, with global travel, the threat of MERS-CoV spreading to our shores is very real, as highlighted by the recent fatal case of MERS-CoV in a Malaysian who had travelled to Saudi Arabia. As a community of infectious diseases physicians and infection control practitioners, we must remain vigilant, and put in place infrastructure and policies to deal with emerging infections and pandemics.

\section{REFERENCES}

1. World Health Organization (WHO). Global Alert and Response (GAR): Middle East respiratory syndrome coronavirus (MERS-CoV)update. 23 April 2014 [online]. Available at: http://www.who.int/csr/ don/2014_04_23_mers/en/. Accessed April 24, 2014.

2. Centers for Disease Control and Prevention. Middle East Respiratory Syndrome (MERS) [online]. Available at: http://www.cdc.gov/coronavirus/ mers/. Accessed April 24, 2014. 
3. World Health Organization (WHO). Global Alert and Response (GAR) Coronavirus infections [online]. Available at: http://www.who.int/csr/don/ archive/disease/coronavirus_infections/en/. Accessed April 24, 2014

4. World Health Organization (WHO). Global Alert and Response (GAR): Middle East respiratory syndrome coronavirus (MERS-CoV)update. 17 April, 2014 [online]. Available at: http://www.who.int/csr/ don/2014_04_17_mers/en/. Accessed April 24, 2014.

5. World Health Organization (WHO). Infection prevention and contro during health care for probable or confirmed cases of novel coronavirus (nCoV) infection: interim guidance, 6 May 2013.

6. Health Protection Agency, United Kingdom. Infection Control Advice Possible or Confirmed MERS-CoV Cases: Version 2.0, 28 June 2013 [online]. Available at: http://www.hpa.org.uk/webc/HPAwebFile/ HPAweb_C/1317136232722. Accessed February 13, 2014.

7. Centers for Disease Control and Prevention. Interim Infection Prevention and Control Recommendations for Hospitalised Patients with Middle Eas Respiratory Syndrome Coronavirus (MERS-CoV)[online]. Available at: http://www.cdc.gov/coronavirus/mers/infection-prevention-control.html. Accessed February 13, 2014

8. Guery B, Poissy J, el Mansouf L, et al; MERS-CoV study group. Clinica features and viral diagnosis of two cases of infection with Middle East Respiratory Syndrome coronavirus: a report of nosocomial transmission. Lancet 2013; 381:2265-72.

9. Assiri A, McGeer A, Perl TM, et al; KSA MERS-CoV Investigation Team. Hospital outbreak of Middle East respiratory syndrome coronavirus. N Engl J Med 2013; 369:407-16.

10. World Health Organization (WHO). Internal travel and health: SARS (Severe Acute Respiratory Syndrome) [online]. Available at: http://www. who.int/ith/diseases/sars/en/index.html. Accessed February 13, 2014.

11. Centers for Disease Control and Prevention. Severe Acute Respiratory Syndrome (SARS) Basic Fact Sheet [online]. Available at: http://www.cdc. gov/sars/about/fs-SARS.html\#spreads. Accessed February 13, 2014.

12. Seto WH, Tsang D, Yung RW, et al; Advisors of Expert SARS group of Hospital Authority. Effectiveness of precautions against droplets and contact in prevention of nosocomial transmission of severe acute respiratory syndrome (SARS). Lancet 2003; 361:1519-20.

13. Ho PL, Tang XP, Seto WH. SARS: hospital infection control and admission strategies. Respirology 2003; 8 Suppl:S41-5.

14. Nickell LA, Crighton EJ, Tracy CS, et al. Psychosocial effects of SARS on hospital staff: survey of a large tertiary care institution. CMAJ 2004; 170:793-8.

15. Love RG. Acceptable breathing resistance for respirator use. J Int Soc Respir Protection 1983; 1:45-66

16. Kao TW. The physiological impact of N95 masks on medical staff [online] Available at: http://clinicaltrials.gov/ct/show/NCT00173017. Accessed February 13, 2014.

17. Lim EC, Seet RC, Lee KH, et al. Headaches and the N95 face-mask amongst healthcare providers. Acta Neurol Scand 2006; 113:199-202.

18. Health Protection Agency (HPA) UK Novel Coronavirus Investigation team. Evidence of person-to-person transmission within a family cluster of novel coronavirus infections, United Kingdom, February 2013. Euro Surveill 2013; 18:20427.

19. Memish ZA, Zumla AI, Al-Hakeem RF, Al-Rabeeah AA, Stephens GM Family cluster of Middle East respiratory syndrome coronavirus infections. N Engl J Med 2013; 368:2487-94.
20. Chan $\mathrm{KH}$, Chan JF, Tse $\mathrm{H}$, et al. Cross-reactive antibodies in convalescent SARS patients' sera against the emerging novel human coronavirus EMC (2012) by both immunofluorescent and neutralizing antibody tests. J Infec $2013 ; 67: 130-40$

21. World Health Organization (WHO). Global Alert and Response (GAR). Update 49 - SARS case fatality ratio, incubation period. 7 May 2003 [online]. Available at: http://www.who.int/csr/sarsarchive/2003_05_07a/ en/. Accessed February 13, 2014.

22. Assiri A, Al-Tawfiq JA, Al-Rabeeah AA, et al. Epidemiological, demographic, and clinical characteristics of 47 cases of Middle East respiratory syndrome coronavirus disease from Saudi Arabia: a descriptive study. Lancet Infect Dis 2013; 13:752-61.

23. Jefferson T, Del Mar C, Dooley L, et al. Physical interventions to interrupt or reduce the spread of respiratory viruses: systematic review. BMJ 2009; 339:b3675.

24. Chen WQ, Ling WH, Lu CY, et al. Which preventive measures might protect health care workers from SARS? BMC Public Health 2009; 9:81.

25. Fowler RA, Guest CB, Lapinsky SE, et al. Transmission of severe acute respiratory syndrome during intubation and mechanical ventilation. Am J Respir Crit Care Med 2004; 169:1198-202.

26. Raboud J, Shigayeva A, McGeer A, et al. Risk factors for SARS transmission from patients requiring intubation: a multicentre investigation in Toronto, Canada. PLoS One 2010; 5:e10717.

27. Scales DC, Green K, Chan AK, et al. Illness in intensive care staff after brief exposure to severe acute respiratory syndrome. Emerg Infect Dis 2003; 9:1205-10.

28. Christian MD, Loutfy M, McDonald LC et al; SARS Investigation Team. Possible SARS coronavirus transmission during cardiopulmonary resuscitation. Emerg Infect Dis 2004; 10:287-93.

29. Dwosh HA, Hong HH, AustGarden D, Herman S, Schabas R. Identification and containment of an outbreak of SARS in a community hospital. CMAJ 2003; 168:1415-20.

30. Society of Healthcare Epidemiology of America (SHEA). Position Statement: Interim guidance on infection control precautions for novel swine-origin influenza H1N1 in healthcare facilities, 10 June 2009.

31. World Health Organization (WHO). Interim guidance on infection prevention and control during health care for confirmed, probable or suspected cases of pandemic (H1N1) 2009 virus infection and influenzalike illnesses, 16 December 2009.

32. Centers for Disease Control and Prevention. Interim guidance on infection control measures for $2009 \mathrm{H} 1 \mathrm{~N} 1$ influenza in healthcare settings, including protection of healthcare personnel [online]. Available at: http://www.cdc. gov/h1n1flu/guidelines_infection_control.htm. Accessed February 13, 2014.

33. The National Academies. IOM recommends N95 respirators to protect health care workers from H1N1 flu. 3 September 2009 [online]. Available at: http://www8.nationalacademies.org/onpinews/newsitem. aspx?RecordID=12748. Accessed February 13, 2014

34. Blachere FM, Lindsley WG, Pearce TA, et al. Measurement of airborne influenza virus in a hospital emergency department. Clin Infect Dis 2009; 48:438-40.

35. Loeb M, Dafoe N, Mahony J, et al. Surgical masks vs. N95 respiratory for preventing influenza among health care workers: a randomised trial. JAMA 2009; 302:1865-71 